\title{
Transverse and Longitudinal Lambda Polarization in Lepton Scattering by Unpolarized Nucleons at HERMES
}

\author{
G. Karyan \\ (on behalf of the HERMES Collaboration) \\ A.I. Alikhanyan National Science Laboratory, 2 A. Alikhanian Brothers Street \\ Yerevan, 0036, Armenia \\ gevkar@mail.desy.de
}

Published 29 February 2016

\begin{abstract}
Transverse and longitudinal polarization of $\Lambda$ hyperons has been studied. The transfere of polarization from high-energy positron to $\Lambda^{0}$ hyperon has been measured. The longitudinal spin transverse coefficient is found to be $D_{L L^{\prime}}^{\Lambda}=0.11 \pm 0.10$ (stat) \pm 0.03 (sys). The transverse polarization of $\Lambda$ and $\bar{\Lambda}$ hyperons has been measured in inclusive quasireal photoproduction process for various target nuclei. The polarization observed is positive for light target nuclei and compartaible with zero for heavy target nuclei.
\end{abstract}

Keywords: Lambda; spin transfere; spontaneous polarization.

PACS numbers: 13.88+e, 13.60.-r, 13.60.Rj, 14.20.Jn, 25.30.Rw

\section{Introduction}

A study of $\Lambda$ polarization, i.e. the polarization of the ligthest hyperon with strange quark content has an important role. Due to its parity violating weak decay it has a self-analyzing power, since the decay protons prefer to follow the spin direction of a parent $\Lambda$. The measurements of the longitudinal spin trasnfer from longitudinally polarized beam leptons to the $\Lambda$ hyperon are sensitive to two unknowns : the spin structure of the $\Lambda$ and the spin-dependent dynamics of the fragmentation process in deep-inelastic scattering. More precisely, when a longitudinally-polarized lepton beam is scattered at high energies from a nucleon target, angular momentum conservation dictates that quarks of a particular spin orientation participate predominantly in the interaction. The outgoing struck quark is thus polarized, and hyperons produced from its fragmentation may "remember" its spin orientation and carry a longitudinal polarization themselves. Formally such a correlation may be expressed in terms of a spin-dependent fragmentation function $G_{1 f}^{\Lambda}(z)$ Ref. 1. This

This is an Open Access article published by World Scientific Publishing Company. It is distributed under the terms of the Creative Commons Attribution 3.0 (CC-BY) License. Further distribution of this work is permitted, provided the original work is properly cited. 
fragmentation function has often appeared in the literature with different symbols, most notably as $\Delta D^{\Lambda}(z)$ Ref. 2. or as $\Delta \hat{q}_{\Lambda}(z)$ Ref. 3 .

The transverse polarization of $\Lambda$ hyperons produced in inclusive unpolarized hadron-nucleon, hadron-nucleus, and nucleus-nucleus collisions at high energies is a well-established phenomenon. A substantial transverse $\Lambda$ polarization was first observed in proton-beryllium collisions at a proton-beam energy of $300 \mathrm{GeV}$ Ref. 4 . The polarization $\left|P_{n}^{\Lambda}\right|$ is essentially independent of the beam momentum; its magnitude rises with $p_{T}$, the momentum component transverse to the beam direction, for $p_{T}$ values up to about $1 \mathrm{GeV}$, where it reaches values of up to $\left|P_{n}^{\Lambda}\right| \approx 0.4$. At fixed $p_{T},\left|P_{n}^{\Lambda}\right|$ rises with the Feynman variable $x_{F}=p_{L}^{*} / p_{L, \text { max }}^{*}$ where $p_{L}^{*}$ is the component of the $\Lambda$ momentum in the beam direction measured in the beam-target center-of-mass system and $p_{L, \max }^{*}$ is its maximal possible value. At higher transverse momenta, $P_{n}^{\Lambda}$ measured in such experiments was found to be similar to that observed in $p-p$ or $p-$ nucleus scattering Ref. 5 . While the transverse polarization of hyperons was studied in detail with hadron beams, very little experimental information about $P_{n}^{\Lambda}$ is available from photo or electroproduction. The $x_{F}$ dependence observed in early measurements Ref. 6 , Ref. 7 was very similar to the one seen in $\pi^{-}-p$ reactions, but the data were of low statistical accuracy.

\section{Experiment}

The $\Lambda$ hyperons were accumulated by the HERMES spectrometer at DESY. In this measurements, the $27.6 \mathrm{GeV}$ longitudinally polarized and unpolarized positron beams are passed through an open-ended tubular storage cell into which polarized or unpolarized target atoms in undiluted gaseous form are continuously injected. The HERMES detector is described in detail in Ref. 8. The scattered positrons and

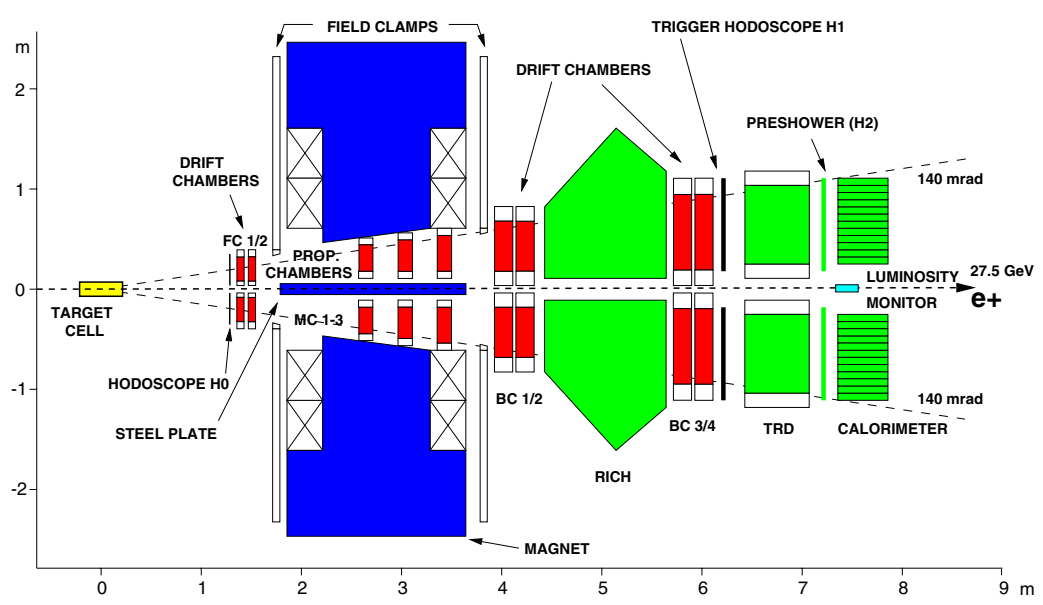

Fig. 1. The HERMES spectrometer. 
the $\Lambda$ decay products were detected by the HERMES spectrometer in the polar angle range from 40 to $220 \mathrm{mrad}$. The $\Lambda^{0}$ hyperons were identified in the analysis through their $p \pi^{-}$decay channel. Events were selected by requiring the presence of at least three reconstructed tracks: a positron track and two hadron candidates of opposite charge or without requiring a positron track for quasireal photoproduction. Track reconstruction was performed with a track fitting algorithm based on the Kalman filter with substantially improved vertex determination and momentum resolution. This algorithm allows for the best possible estimates on track parameters at the beam crossing and/or at the (possible) vertices with other tracks of a given event. The resulting invariant mass distributions for the combined hydrogen and deuterium $(\mathrm{H}+\mathrm{D})$ and the combined krypton and xenon $(\mathrm{Kr}+\mathrm{Xe})$ data are shown in Fig. 2. They were fitted by a Gaussian plus a second-order polynomial line shape. The position of the $\Lambda$ peak agrees within $\sim 0.10 \mathrm{MeV}$ with the world average of $(1115.684 \pm 0.006) \mathrm{MeV}$ Ref. 9. Events within an invariant mass window of \pm $3.3 \sigma$ around the mean value of the Gaussian fit were selected. The fraction $\eta=$ $N^{\Lambda} /\left(N^{\Lambda}+N^{b g r}\right)$ of $\Lambda$ events in the selected mass window is about $\sim 96 \%$ for all targets.
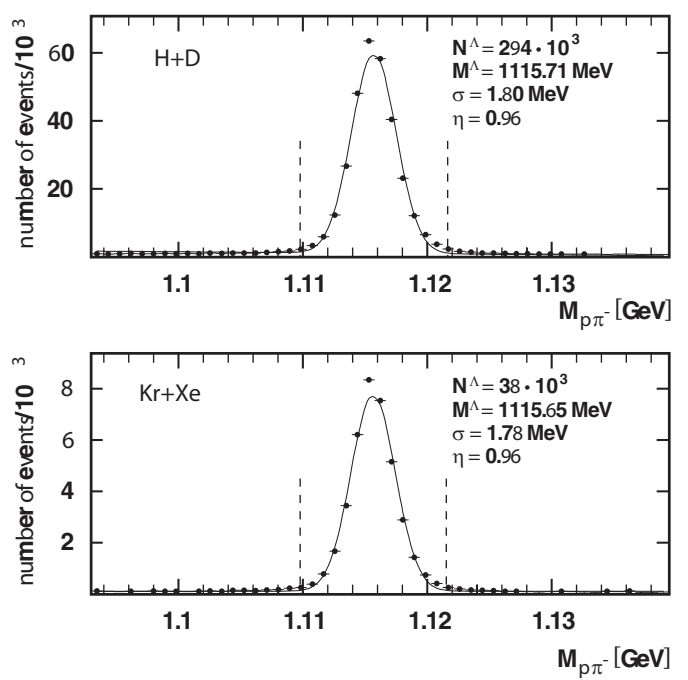

Fig. 2. Invariant-mass distributions for $\Lambda$ events obtained with hydrogen and deuterium targets (top panel) and with krypton and xenon targets (bottom panel). The vertical lines indicate the invariant-mass interval used for the determination of the $\Lambda$ polarization. The quantities given in the legends are the number of analyzed $\Lambda$ events, $N^{\Lambda}$, in the selected invariant-mass window after subtraction of background events, the reconstructed $\Lambda$ mass $M^{\Lambda}$, the resolution of the invariant-mass distribution, and the fraction $\eta$ of $\Lambda$ events in this mass window. 


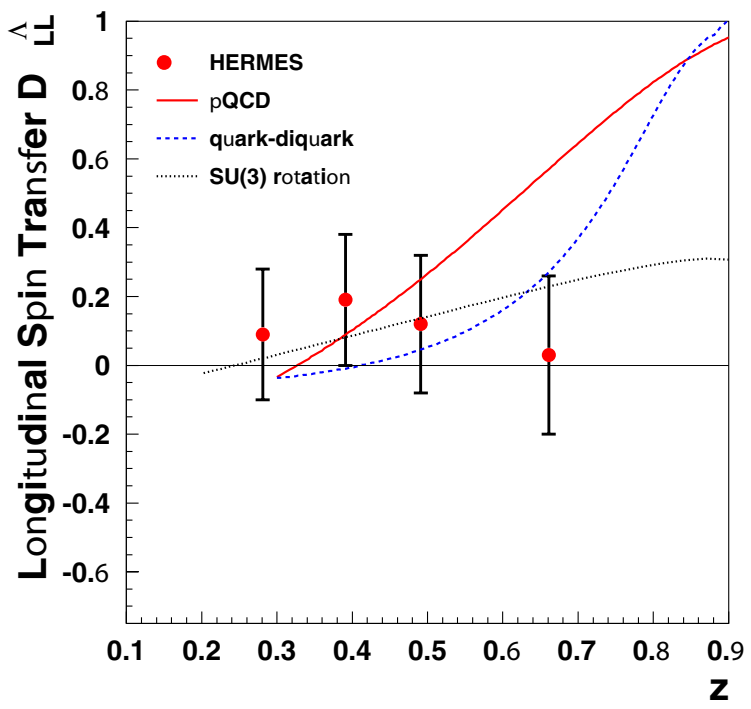

Fig. 3. Dependence of the longitudinal spin-transfer coefficient $D_{L L^{\prime}}^{\Lambda}$ on $z$, for $x_{F}>0$. The curves represent the phenomenological model calculations. Error bars are statistical only.

\section{Results}

The polarization transfer $D_{L L^{\prime}}^{\Lambda}$ from a polarized beam positron to a semi-inclusively produced $\Lambda^{0}$ hyperon has been measured in deep-inelastic positron scattering off various unpolarized targets Ref. 10. The spin transfer for the background $D_{L L^{\prime}, b g r}^{\Lambda}$ was determined from the events above and below the peak outside of the $3.3 \sigma$ invariant mass window. In order to obtain the final result for the net $\Lambda^{0}$ events, the spin transfer within the $\Lambda^{0}$ peak was corrected for this background contribution in each kinematic bin as

$$
D_{L L^{\prime}}^{\Lambda}=\frac{\hat{D}_{L L^{\prime}}^{\Lambda}-\epsilon D_{L L^{\prime}, b g r}^{\Lambda}}{1-\epsilon} .
$$

where $\epsilon=N_{b g r} /\left(N_{\Lambda}+N_{b g r}\right)$. The overall systematic uncertainty of the measured spin transfer is estimated to be \pm 0.03 . The result for the spin transfer coefficient $D_{L L^{\prime}}^{\Lambda}$ is presented in Fig. 3 .

The transverse $\Lambda$ polarization $P_{n}^{\Lambda}$ was measured in inclusive quasireal photoproduction on various nuclei Ref. 11. The systematic uncertainty of $P_{n}^{\Lambda}$ has been estimated to be \pm 0.02 . This value was derived from detailed Monte Carlo studies that took into account possible detector misalignments, and also from the false polarization measured for $K_{s}^{0} \rightarrow \pi^{+} \pi^{-}$events that provide an event topology with two separated vertices similar to $\Lambda$ decays. The experimental results for the extracted transverse polarization $P_{n}^{\Lambda}$ as a function of the atomic-mass number $A$ are shown in Fig. 4. 


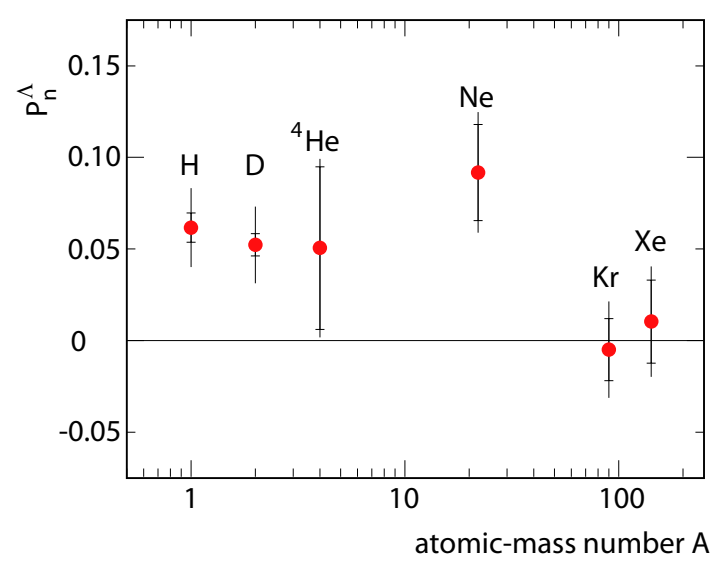

Fig. 4. Dependence of the transverse polarization $P_{n}^{\Lambda}$ on the atomic-mass number A of the target nuclei. The inner error bars represent the statistical uncertainties; the full error bars represent the total uncertainties, evaluated as the sum in quadrature of statistical and systematic uncertainties.

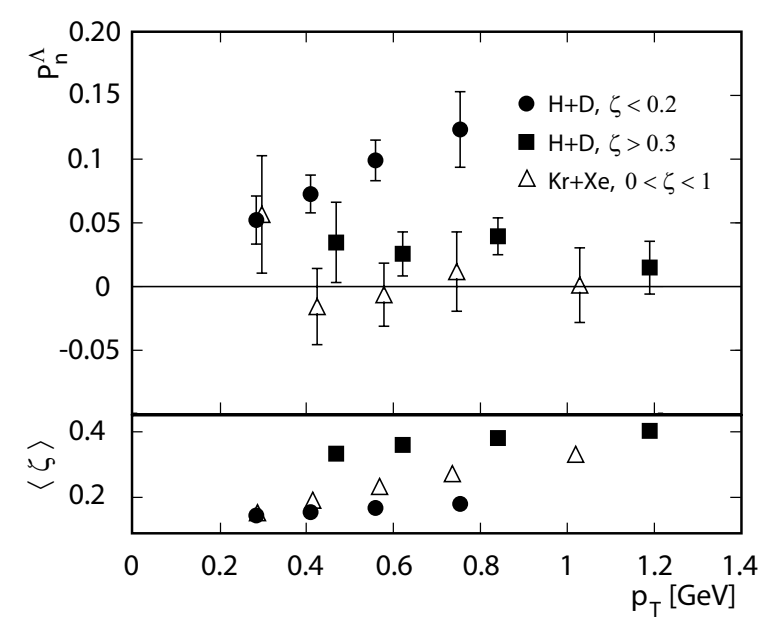

Fig. 5. Dependence of the transverse polarization $P_{n}^{\Lambda}$ on the transverse $\Lambda$ momentum $p_{T}$. Closed circles (squares) represent the combined hydrogen and deuterium data for the region $\zeta<0.2$ ( $\zeta$ $>0.3$ ). The combined krypton and xenon data (open triangles) are shown for the full range. The error bars represent the statistical uncertainty. The values of $\zeta$ for each $p_{T}$ bin are shown in the lower panel.

In Fig. 5 the transverse polarization is shown as a function of $p_{T}$.

The $\mathrm{H}+\mathrm{D}$ data are presented for two intervals in the variable $\zeta\left(\zeta=\left(E^{\Lambda}+\right.\right.$ $\left.p_{L}\right) /(E+k) \approx E^{\Lambda} / E$, here $E^{\Lambda}$ and $E$ are energies of the produced $\Lambda$ and beam lepton respectively ). The $p_{T}$ dependence in these two intervals is rather different. In the 
region $\zeta<0.2$, where the produced hyperons mainly stem from the backward region, the polarization increases linearly with $p_{T}$ up to a value of $\sim 0.12$ at $p_{T} \simeq 0.75$ $\mathrm{GeV}$ (closed circles), while in the region $\zeta>0.3$ (closed squares) the polarization is substantially smaller with very little dependence on $p_{T}$.

\section{References}

1. P.J. Mulders and R.D. Tangerman, Nucl. Phys. B, 461, 197 (1996).

2. B.-Q. Ma, J.-J. Yang, I. Schmidt, Phys. Lett. B, 477, 107 (2000).

3. R.L. Jaffe, Phys. Rev. D, 54, 6581 (1996).

4. G. Bunce et al., Phys. Rev. Lett., 36, 1113 (1976).

5. R. Bellwied [for the E896 Collaboration], Nucl. Phys. A, 698, 499c (2002).

6. D. Aston et al. (WA004 Collaboration), Nucl. Phys. B, 195, 189 (1982).

7. K. Abe et al. (BC072 Collaboration), Phys. Rev. D, 29, 1877 (1984).

8. HERMES Collaboration, K. Ackerstaff et al., Nucl. Instrum. Methods A, 417, 230, (1988).

9. J. Beringer et al., Particle Data Group, Phys. Rev. D, 86, 010001, (2012).

10. A. Airapetian et al. (HERMES Collaboration), Phys. Rev. D, 74, 072004, (2006).

11. A. Airapetian et al. (HERMES Collaboration), Phys. Rev. D, 90, 072007, (2014). 